SUBJECT AREAS: MATERIALS CHEMISTRY NANOSCALE MATERIALS

Received

19 September 2014

Accepted

27 November 2014

Published

13 January 2015

Correspondence and requests for materials should be addressed to Z.H. (zhenguo@uow. edu.au)

\section{Synthesis of Large and Few Atomic Layers of Hexagonal Boron Nitride on Melted Copper}

\author{
Majharul Haque Khan', Zhenguo Huang', Feng Xiao', Gilberto Casillas' ${ }^{2}$, Zhixin Chen ${ }^{3}$, Paul J. Molino ${ }^{4}$ \\ \& Hua Kun Liu'
}

${ }^{1}$ Institute for Superconducting and Electronic Materials, University of Wollongong, Wollongong, NSW 2500, Australia, ${ }^{2}$ Electron Microscopy Center, University of Wollongong, Wollongong, NSW 2500, Australia, ${ }^{3}$ Bluescope Steel Metallurgical Center, University of Wollongong, Wollongong, NSW 2500, Australia, ${ }^{4}$ Intelligent Polymer Research Institute, University of Wollongong, Wollongong, NSW 2500, Australia.

Hexagonal boron nitride nanosheets $(h$-BNNS) have been proposed as an ideal substrate for graphene-based electronic devices, but the synthesis of large and homogeneous $h$-BNNS is still challenging. In this contribution, we report a facile synthesis of few-layer $h$-BNNS on melted copper via an atmospheric pressure chemical vapor deposition process. Comparative studies confirm the advantage of using melted copper over solid copper as a catalyst substrate. The former leads to the formation of single crystalline $\boldsymbol{h}$-BNNS that is several microns in size and mostly in mono- and bi-layer forms, in contrast to the polycrystalline and mixed multiple layers (1-10) yielded by the latter. This difference is likely to be due to the significantly reduced and uniformly distributed nucleation sites on the smooth melted surface, in contrast to the large amounts of unevenly distributed nucleation sites that are associated with grain boundaries and other defects on the solid surface. This synthesis is expected to contribute to the development of large-scale manufacturing of $h$-BNNS/graphene-based electronics.

S imilar to carbon, boron nitride commonly exists in hexagonal ( $h$-BN) and cubic forms (c-BN), where the former features the $s p^{2}$ and the latter adopts the $s p^{3}$ hybridization configuration. $h$-BN features a layered structure where the $\mathrm{B}$ and $\mathrm{N}$ atoms form hexagonal honeycombs within a layer and the layers are held together via van der Waals forces, with $\mathrm{N}$ atoms on top of $\mathrm{B}$ atoms and vice versa to construct the $h$-BN lattice. $h$ $\mathrm{BN}$ and its carbon counterpart, graphite, have very similar bond lengths and interlayer distances. Atomic layered $h$-BN, i.e., nanosheets ( $h$-BNNS), is also called 'white graphene', since it is white with a large direct band gap of 5.0 to $6.0 \mathrm{eV}^{1-4}$, in contrast to graphene's zero band gap that results in a black color. $h$-BNNS has shown some exceptional properties that lend themselves to applications different from those of graphene. For instance, $h$ BNNS is oxidation resistant up to $1000^{\circ} \mathrm{C}$ in air ${ }^{5}$, while graphene deteriorates around $600^{\circ} \mathrm{C}^{6} . h$-BNNS is highly resistant to wet chemical attack and is difficult to functionalize ${ }^{7}$, while graphene readily turns into graphene oxide in a mixed sulfuric acid, sodium nitrate, and potassium permanganate solution ${ }^{8}$. Owing to its ultra-flat surface, absence of dangling bonds, and iso-structure with graphene, $h$-BNNS has been reported to be a better substrate than silicon with respect to charge transport through graphene, thereby opening up a large potential for application in future graphene-based devices 9 . $h$-BNNS has been utilized as a dielectric layer for the fabrication of graphene-based field-effect transistors ${ }^{10-12}$ and explored as a tunneling barrier within graphene layers ${ }^{13}$. For these applications, high quality $h$-BNNS that is large in size, uniform in thickness, and free of defects, is highly critical. The synthesis of such high quality $h$-BNNS, however, remains challenging.

Various techniques have been explored to synthesize $h$-BNNS, including mechanical cleavage ${ }^{14}$, liquid phase exfoliation ${ }^{15}$, unwrapping $\mathrm{BN}$ nanotubes ${ }^{16}$, substitution reaction ${ }^{17}$, radio frequency magnetron sputtering ${ }^{18}$, electron beam irradiation ${ }^{19}$, and chemical vapor deposition $(\mathrm{CVD})^{20-28}$. Among these, the CVD method has been established as a popular route to synthesize large nanosheets. Mono-layer $h$-BNNS was produced as early as 1995 using an ultra-high vacuum (UHV) CVD system ${ }^{20}$. Recently, simple atmospheric pressure CVD (APCVD $)^{21-24}$ and low pressure CVD (LPCVD) ${ }^{25-27}$ systems have been developed to grow large $h$-BNNS. Polycrystalline copper and nickel foils have been routinely used as substrates/catalysts in CVD systems because of their wide availability, low cost, and ease of etching to facilitate the transfer of $h$-BNNS onto different substrates. As far as conventional polycrystalline foils are concerned, however, the CVD method is not free from limitations. Poor controllability 
over the $h$-BNNS thickness is one of the primary drawbacks ${ }^{19,25}$. In the CVD method, nucleation of $h$-BN has been observed along the crystallographic defects of the polycrystalline catalyst surface, which consequently reduces the quality of the final product ${ }^{24,26}$. Single crystal foil can be an option to remove the grain boundary induced nucleation sites ${ }^{20,29,30}$; however, the high cost and small size of single crystal foils limit their use. It has been demonstrated that improving the surface smoothness by employing techniques such as electropolishing is conducive to the formation of large domains of $h$ $\mathrm{BNNS}^{24}$. Melting can lead to an ultra-flat liquid surface that is free of grain boundaries and crystallographic defects, and has been found to facilitate the growth of large uniform graphene single crystal ${ }^{31,32}$. In this paper, we report melted-copper facilitated APCVD growth of large and few-layer $h$-BNNS. For comparison, the growth was also carried out on solid copper foil at $1000^{\circ} \mathrm{C}$ under similar conditions. The $h$-BNNS samples were characterized using X-ray photoelectron spectroscopy (XPS), atomic force microscopy (AFM), Raman spectroscopy, scanning electron microscopy (SEM), and transmission electron microscopy (TEM).

\section{Results}

$h$-BNNS was grown on solid and melted copper substrates in a split type tube furnace (see details in the Methods section and Supplementary Information). For simplicity, $h$-BNNS grown on solid and melted copper substrates are denoted hereafter as S- $h$ BNNS and M- $h$-BNNS, respectively. To determine the crystallinity, size, and thickness of the nanosheets, both S- $h$-BNNS and M- $h$ BNNS were transferred to $285 \mathrm{~nm} \mathrm{SiO} 2$ coated $\mathrm{Si}$ wafers and holey carbon TEM grids (see details in the Methods section).

Clear optical contrast was observed between S- $h$-BNNS and M- $h$ $\mathrm{BNNS}$ on the $\mathrm{SiO}_{2} / \mathrm{Si}$ wafers. The clear blue region in Fig. $1 \mathrm{a}$ and the nearly transparent area indicated by the dotted line in Fig. 1b represent thick and thin $h$-BNNS grown on the solid and melted copper substrates, respectively ${ }^{33}$. The thickness of $h$-BNNS on $\mathrm{SiO}_{2} / \mathrm{Si}$ wafer was measured by AFM (Figs. $1 \mathrm{c}$ and d). A thickness of $4.2 \mathrm{~nm}$ was observed for the S- $h$-BNNS, indicating $\sim 12$ layer stacking. In contrast, $\mathrm{M}$ - $h$-BNNS is only $1.0 \mathrm{~nm}$ thick, corresponding to $1-3$ atomic layers. It should be noted that underlying trapped water or contamination between the $h$-BNNS and the $\mathrm{SiO}_{2}$ substrate can often increase the thickness under AFM, and therefore, $1 \mathrm{~nm}$ corresponds to the height of a mono-layer of $h$ - $\mathrm{BN}^{33,34}$. The AFM thickness analysis results were further corroborated by high resolution (HR) TEM studies, which will be discussed later.

XPS was employed to assess the stoichiometry and nature of the bonding between $\mathrm{B}$ and $\mathrm{N}$ atoms (Figs. 1e, $\mathrm{f}$ and Fig. $\mathrm{S} 1$ in Supplementary Information]. For both samples, the B 1 s and N 1s peaks are situated at 189.8-190.9 eV and 397.1-398.7 eV, respectively, which match the published results ${ }^{21,24,25,27}$. The $\mathrm{B} / \mathrm{N}$ atomic ratio was estimated based upon the integrated peak areas and the peak sensitivity factors. The $\mathrm{B} / \mathrm{N}$ atomic ratio is found to be within the $1: 1.04-1: 1.12$ range, close to the $1: 1$ stoichiometric ratio for $s p^{2}$ bonded $h-\mathrm{BN}$.

Raman spectra of $h$-BNNS were obtained on a Renishaw InVia Raman spectrophotometer using a $514.5 \mathrm{~nm} \mathrm{Ar}^{+}$laser source. Figure $1 \mathrm{~g}$ shows the typical B-N stretching vibration mode $\left(\mathrm{E}_{2 \mathrm{~g}}\right)$ of $h$ - $\mathrm{BN}$ on $\mathrm{SiO}_{2} / \mathrm{Si}$ substrate ${ }^{33,35}$. The lower intensity of the $\mathrm{E}_{2 \mathrm{~g}}$ peak of $\mathrm{M}-h$-BNNS reflects its stacking of fewer atomic layers in comparison to $\mathrm{S}-h$-BNNS ${ }^{21,23,33,35}$. A peak shift of $3 \mathrm{~cm}^{-1}$ is also observed for M- $h$ BNNS. In general, shifting in $\mathrm{E}_{2 \mathrm{~g}}$ can occur under different strain conditions within the layers: a compressive strain results in a blue shift and a tensile strain in a red shift ${ }^{21,25,33}$. For mono-layer $h$-BNNS, several reasons have been proposed for the large blue shift, including hardening of the $\mathrm{E}_{2 \mathrm{~g}}$ phonon due to the shorter $\mathrm{B}-\mathrm{N}$ bonds in the mono-layer ${ }^{33}$, intrinsic wrinkles due to the substrate surface ${ }^{21}$, doping effects ${ }^{33,36}$, and lack of interaction between neighboring layers ${ }^{33}$. The blue shift in the M- $h$-BNNS in our case is likely to be associated with compressive strain and wrinkle formation. Thermal expansion mismatch between the $h$-BNNS and the copper occurs during cooling, where copper contracts and $h$-BNNS expands, giving rise to a compressive strain on the sheets ${ }^{37}$. For the S- $h$-BNNS, the shift in $\mathrm{E}_{2 \mathrm{~g}}$ is negligible. It is reasonable to assume that less compressive strain exists in S- $h$-BNNS, since the thermal contraction of the solid copper substrate is much less than that of the melted copper. In addition, thick S- $h$-BNNS is less susceptible to the formation of wrinkles.

Figure $1 \mathrm{~h}$ shows a high angle annular dark field scanning TEM (HAADF-STEM) image of a bi-layer of $h$-BNNS obtained at $80 \mathrm{kV}$. The fast Fourier transform (FFT) obtained from the whole image shows one group of distinct hexagonal symmetric spots, which indicates a highly periodic $h$-BN structure with no differences in rotational orientation between the layers. The darker and brighter regions in Fig. 1h have been identified as mono- and bi-layer $h$ BNNS, respectively ${ }^{5}$. The mono-layer region was created by removing the $\mathrm{B}$ and $\mathrm{N}$ atoms of the top layer with an electron beam (see details in Supplementary Information Fig. S2). The FFT filtered B mono-vacancy image (inset enclosed by the red square in Fig. 1h) clearly indicates a missing $\mathrm{B}$ atom. The brighter spots are identified as $\mathrm{N}$ atoms, due to their slightly higher atomic number than $\mathrm{B}$ atoms, causing Z-contrast intensity variation in the HAADF images ${ }^{38}$. This has also enabled direct observation of $\mathrm{B}$ and $\mathrm{N}$ atoms in a hexagonal network in a FFT filtered HAADF-STEM image (Fig. 1i) (see the raw HAADF-STEM image in Supplementary Information Fig. S3). The line profile along the trace in Fig. 1i confirms that the $s p^{2}$ bonded B and $\mathrm{N}$ form a hexagonal honeycomb structure in the mono-layer $h$ $\mathrm{BN}$, while the presence of other elements would have been identified from the relative intensity difference ${ }^{38}$.

Electron energy loss spectroscopy (EELS) was conducted to investigate the elemental composition and nature of the bonding in the $h$-BNNS. The EELS spectrum (Fig. 1k) confirms the presence of characteristic B K edge and $\mathrm{N} K$ edge peaks of $h$-BN, starting at $195 \mathrm{eV}$ and $406 \mathrm{eV}$, respectively ${ }^{21,39}$. The atomic ratio of $\mathrm{B} / \mathrm{N}$ derived from the EELS spectrum is $1: 1.16$, close to the number based upon the XPS results. It should be noted that this ratio can have an error of up to $12.2 \%$ due to uncertainty in the baseline determination ${ }^{39}$. The presence of $1 \mathrm{~s}-\rightarrow \pi^{*}$ and $1 \mathrm{~s} \rightarrow \sigma^{*}$ anti-bonding orbitals next to B K and $\mathrm{N} K$ shell ionization edges confirms the formation of $s p^{2}$ bonded $h$-BN ${ }^{10,21,35,39}$. The small C K edge near $289 \mathrm{eV}$ is from the residual poly(methyl methacrylate) (PMMA) on $h$-BNNS ${ }^{10,21}$.

An aberration corrected STEM study was conducted to confirm the formation of large single crystalline $h$-BNNS growth on the melted copper. Figure 2a shows a low magnification HAADFSTEM image of the M- $h$-BNNS. Selected area electron diffraction (SAED) patterns were acquired from the specified regions circled in red in Fig. 2a. One set of discrete six-fold symmetry patterns was obtained, with less than $1^{\circ}$ rotational orientation difference between the patterns, suggesting $\sim 4 \mu \mathrm{m}^{2}$ single crystalline $h$-BNNS in that region. The dark areas in the image are voids, where the nanosheet was broken during the transfer process. A scrolled up nanosheet edge is identified in (a) by the green arrow. Some of the sheets were folded, which facilitates the quantification of the $h$-BNNS layers in the bright field (BF) STEM imaging mode. Figure $2 b$ and $c$ show the edges of mono- and bi-layer $h$-BNNS, respectively. A statistical distribution of the $h$-BNNS layers from the analysis of 40 STEM images from different regions is shown in Fig. 2 d. Around $90 \%$ of the $h$-BNNS are found to be mono- and bi-layer on melted copper substrate.

For S- $h$-BNNS, $1-10$ layers were observed within a micron-sized sheet, which indicates largely inhomogeneous $h$-BNNS growth on the solid copper substrate (Fig. 3 and Supplementary information Fig. S5). The SAED patterns show several sets of hexagonal patterns, indicating the polycrystalline nature of the sheets within the SAED zone $\left(800 \mathrm{~nm}^{2}\right)^{40,41}$.

\section{Discussion}

There are several factors proposed as responsible for the observed differences in $h$-BNNS morphology and crystallinity when the 

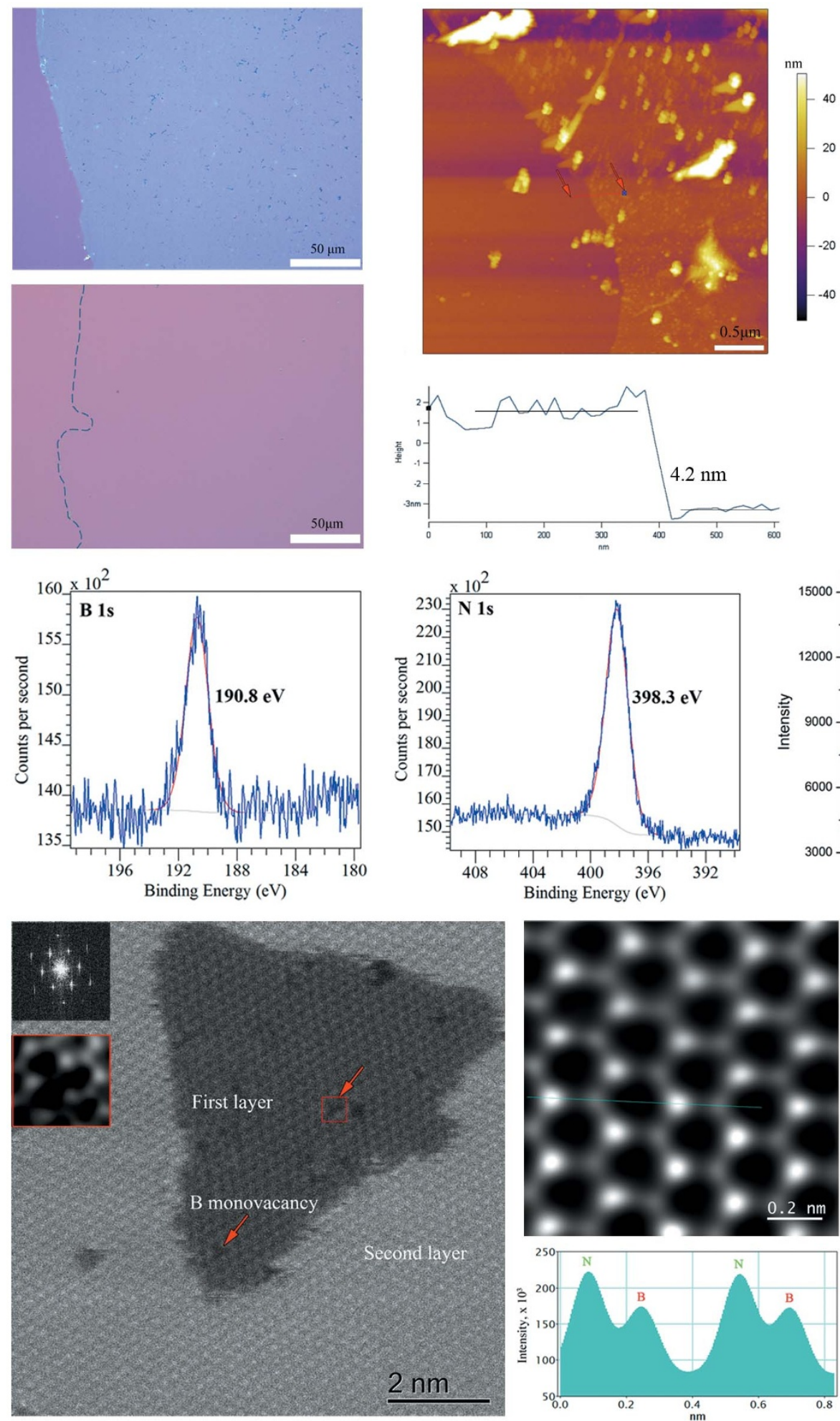
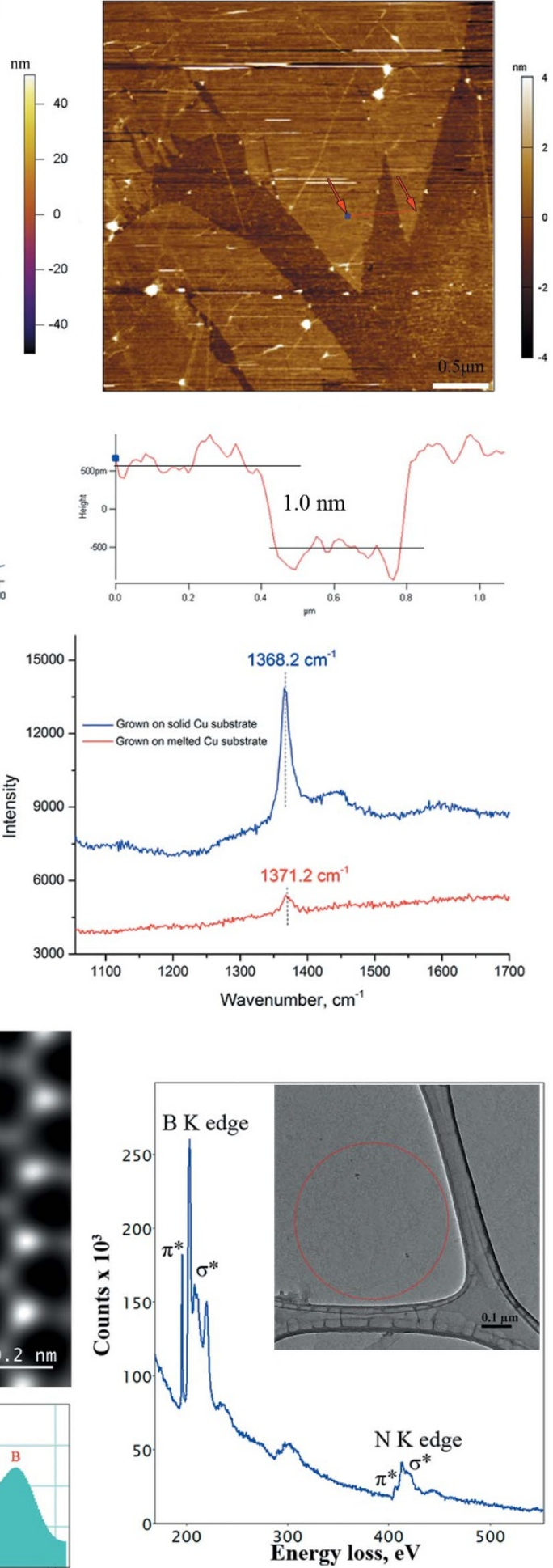

Figure 1 Characterization of S-h-BNNS and M-h-BNNS. (a), (b) Optical images; (c), (d) AFM height images with corresponding thickness profiles on $\mathrm{SiO} 2 / \mathrm{Si}$ wafer for the S-h-BNNS and M-h-BNNS samples, respectively. (e), (f) Representative B 1s and N 1s core level peaks in XPS spectra.

(g) Comparative Raman spectra of h-BNNS grown on solid and melted copper substrates and then transferred to SiO2/Si substrate. (h) HAADF-STEM image of a bi-layer h-BNNS. The dark triangular region is mono-layer h-BN, which was exposed after electron beam radiation removed the top layer. The FFT pattern in the top inset shows a distinct six-fold hexagonal pattern, characteristic of h-BN. The inset in the red square shows a FFT filtered B monovacancy zone. (i) FFT filtered HAADF-STEM image of a defect-free h-BN mono-layer region. (j) Line profile along the trace in (i). (k) EELS spectrum acquired from the circled region in the inset image, showing the $\mathrm{K}$ shell ionization edges of $\mathrm{B}$ and $\mathrm{N}$, which confirms the $\mathrm{sp} 2 \mathrm{bonded} \mathrm{h}-\mathrm{BN}$ formation. The $\mathrm{C}$ peak possibly comes from the PMMA residues after the transfer. 

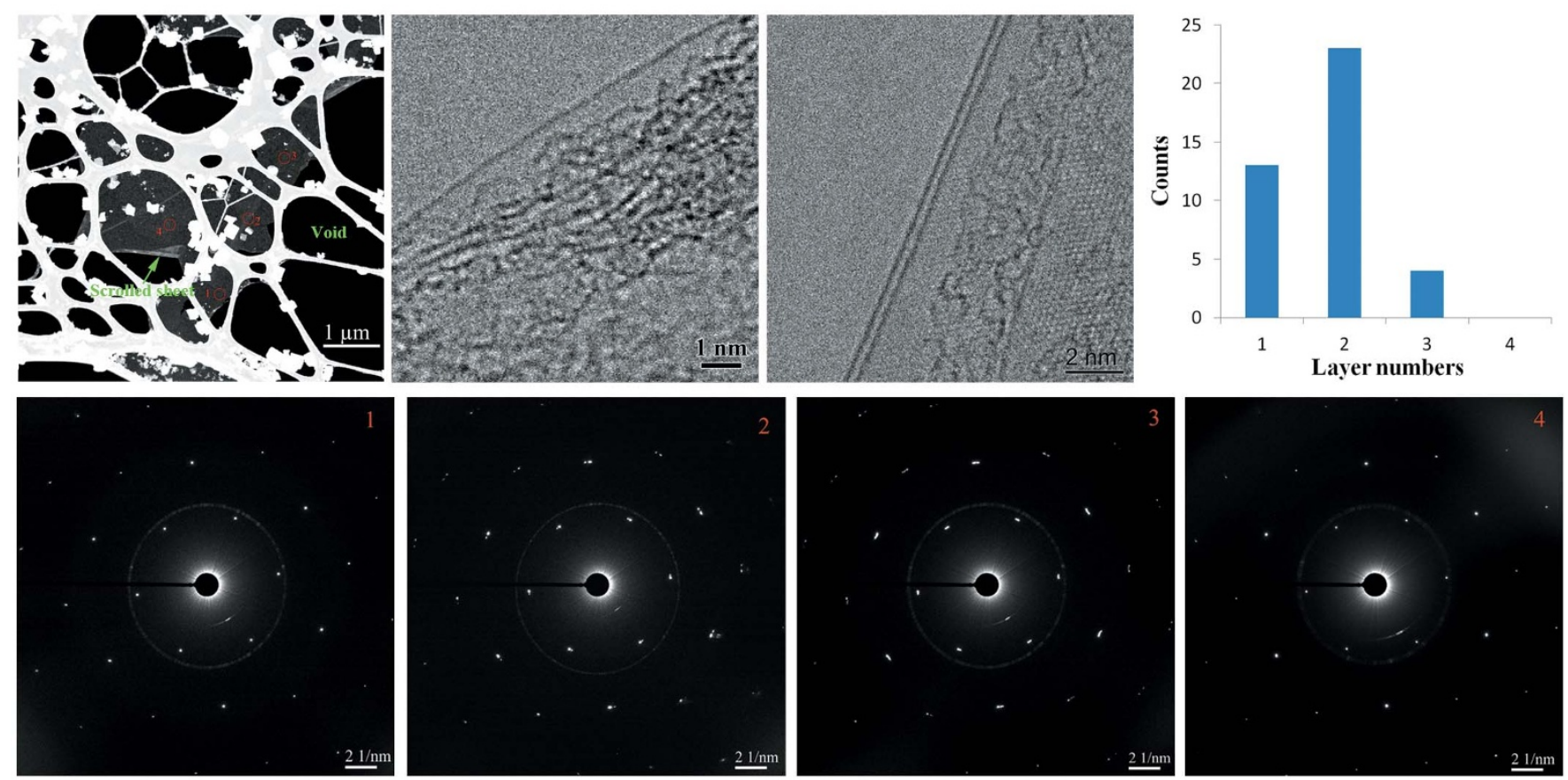

Figure 2 HAADF-STEM characterization of M-h-BNNS. (a) Low magnification HAADF-STEM image of M-h-BNNS. SAED patterns (bottom) were obtained from the four circled areas. The distinctive set of hexagonal spots in the SAED patterns indicates the single crystalline nature of the nanosheet within that region. The difference in rotational orientation in the four SAED patterns is less than $1^{\circ}$. BF-STEM images of (b) mono- and (c) bilayer h-BNNS. (d) Statistical distribution of h-BNNS atomic layers based upon 40 BF-STEM images.

nanosheets are grown on the solid and melted copper. One key factor in growing large single crystalline $h$-BNNS is reducing the number of nucleation sites at the beginning of growth ${ }^{24}$, which has also been found to be critical for the CVD synthesis of large crystalline graphene ${ }^{42,43}$. Due to the high interfacial energy along the defects (grain boundaries, rough edges, defect lines, impurities) on the solid copper surface, the activation energy of $h$-BN nucleation is lower at those sites than within the smooth regions. This phenomenon can be clearly observed when the growth is carried out using a limited amount of ammonia borane. Triangular $h$-BN domains are seen to grow preferentially along the manufacturing-induced parallel defect lines and grain boundaries on the solid copper surface (Figs. 4a, b and Supplementary Information Fig. S6). At the nucleation center of each triangular domain, minute triangular $h$-BN adlayers are clearly noticeable (inset in Fig. 4a) (Numbers of layers are identifiable based upon the contrast in the SEM images ${ }^{44}$.) $h$-BN adlayers are also observed along the merging areas (inset in Fig. 4c). Adlayers have been observed during the CVD synthesis of graphene $e^{45}$. It has been proposed that carbon atoms diffuse between the copper and the existing monolayer, reach the center, and then form the secondary adlayers. This is unlikely the case for $h$-BNNS, however, since the diffusion of the $\mathrm{BN}$ monomers would encounter much resistance due to their size. The formation of adlayers is associated with the defects, both at the center and at grain boundaries, of existing $\mathrm{h}-\mathrm{BN}$ monolayer. The nucleation sites associated with substrate defects would cause strain in the existing monolayer, particularly prominent at the center, which favors further nucleation on top of the existing layer, thus leading to multiple $h$-BNNS on a solid copper surface. When more ammonia borane (10-20 mg) is used, these triangular domains grow in the lateral direction and join up with the neighboring ones (Fig. 4c). Along the joining lines, secondary $h$-BN nucleation is observed. Further increasing the amount of ammonia borane (i.e., $100 \mathrm{mg}$ ) results in complete coverage of the copper surface, but with various small triangular $h-\mathrm{BN}$ islands on the top (Fig. $4 \mathrm{~d}$ ).

The coalescence of these domains occurs by the edge attachment of new diffused $h$-BN monomers. The diffusion rate of these monomers is limited on the solid copper surface at $1000^{\circ} \mathrm{C}$ in comparison to $1100^{\circ} \mathrm{C}$ for melted copper. The movement of these monomers is
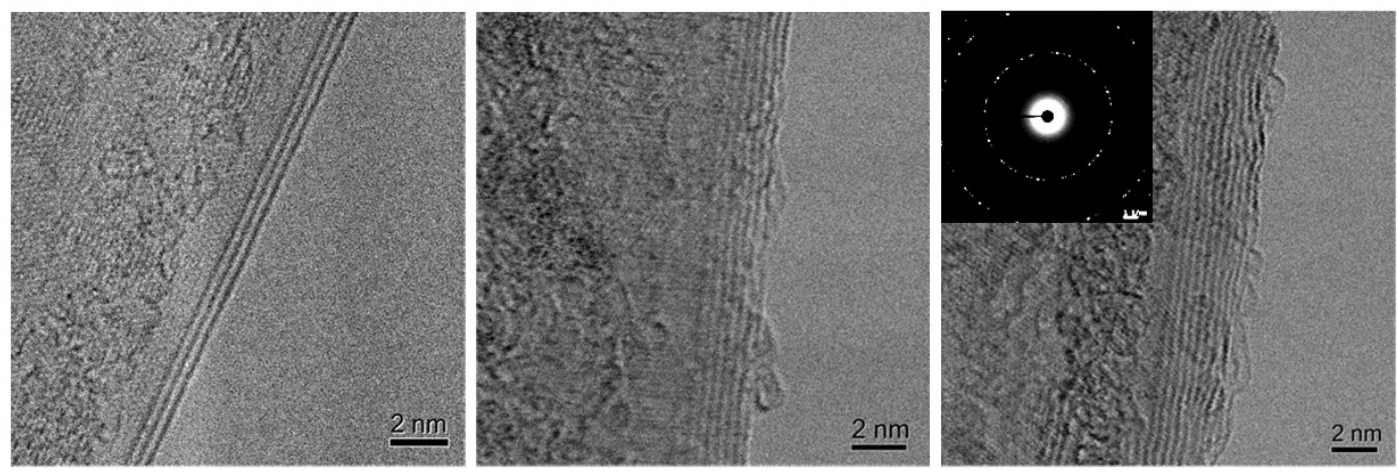

Figure 3 BF-STEM images of S-h-BNNS. (a) 3-layer, (b) 7-layer, and (c) 10-layer h-BNNS. A representative SAED pattern of the S-h-BNNS is shown in the inset of to (c). 

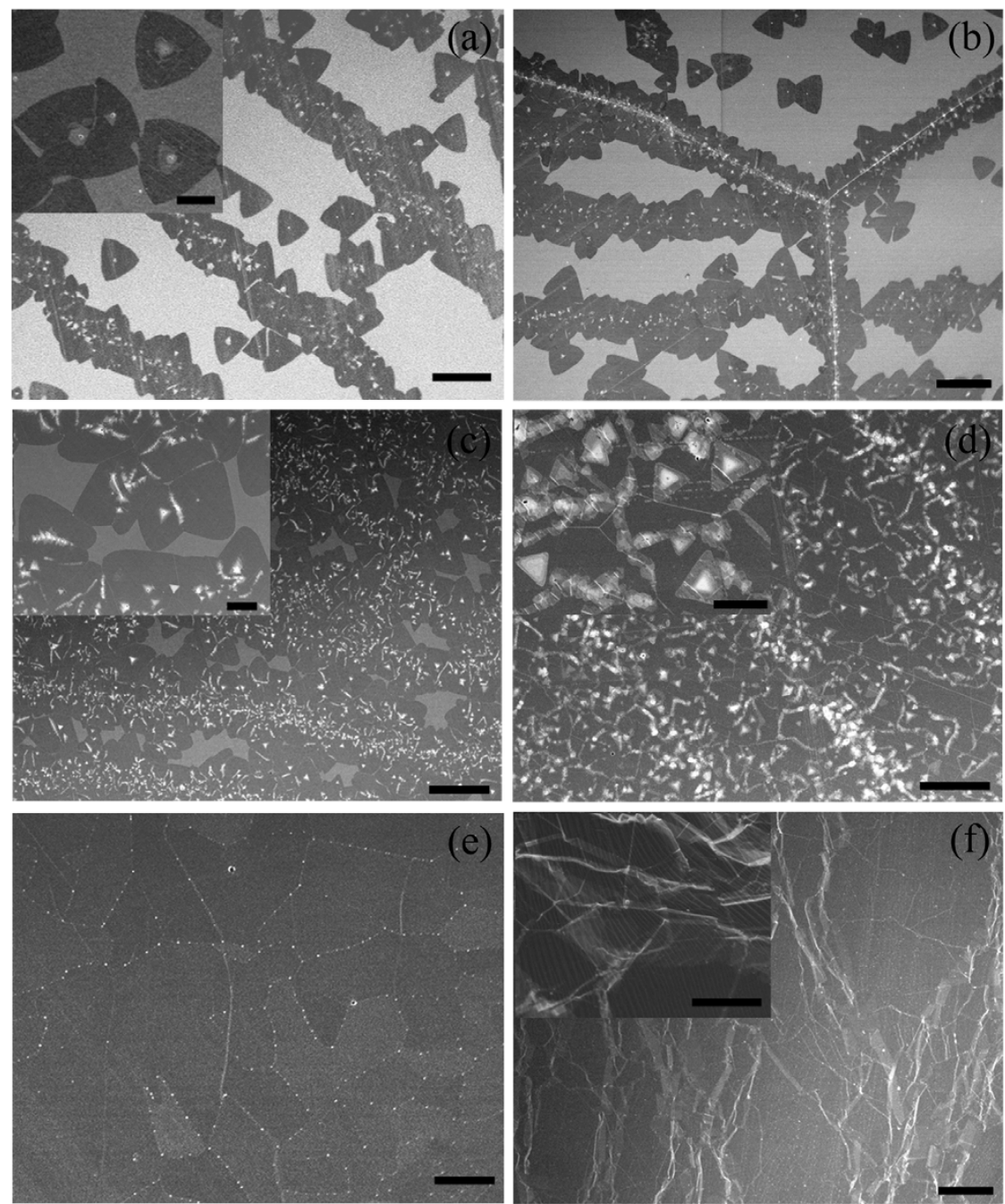

Figure 4 SEM images S-h-BNNS and M-h-BNNS at different growth stage, with the insets showing corresponding images at higher magnification. For solid copper, (a), (b) initial h-BN triangular domains with adlayers at the nucleation centers grow preferentially along the defect lines and grain boundaries. (c), (d) more ammonia borane results in new h-BN domains, while the initial triangular domains grow in the lateral direction and coalesce with their neighbors to form a continuous sheet. For melted copper, (e), (f) initial h-BN domains grow randomly on the surface due to the absence of defect lines and grain boundaries. Increasing the amount of ammonia borane leads to a complete coverage. For the whole process, no adlayer growth is observed. The scale bars are $5 \mu \mathrm{m}$ in the images and $1 \mu \mathrm{m}$ in the insets.

also inhibited by the defect lines on the surface. As a result, the crystal size (based on SAED, more in Supplementary Information Fig. S5) of the $h$-BNNS on solid copper is found to be much smaller than that on the melted surface. Due to the effective catalyst contact, the $h$-BN growth rate of the first layer exceeds that of the adlayers. The island shaped domains grow in the lateral direction in the presence of sufficient borazine and ultimately join up with the neighboring domains. This leads to a large $h$-BNNS with a large number of adlayers attached to the sheet. Accordingly, S- $h$-BNNS growth follows the Volmer-Weber island model ${ }^{46}$.

Melting the copper eliminates grain boundaries and other high energy defects, which then effectively reduces the number of nucleation sites. The $h$-BN nucleation starts with the supersaturation of the borazine molecules ${ }^{47}$ over random locations on the melted surface. The initial $h$-BN domains are mono-layer in nature, with no observable adlayers on top (Fig. 4e). After the initial $h$-BN nucleation, the smooth defect-free melted copper surface provides a lower kinetic energy barrier and longer diffusion distance for the $h$-BN monomers to travel, adjust, and attach themselves to the existing nuclei in an oriented way. Such a growth process can therefore lead to the formation of large single crystalline $h$-BNNS. Increasing the amount of ammonia borane $(100 \mathrm{mg})$ causes the single layers to join together, and finally, a large continous and wrinkled nanosheet is obtained (Fig. $4 \mathrm{f}$ ). The presence of up to 3 layers $(<10 \%)$ of $h$-BNNS on the melted copper suggests that the growth is not surface-limited. The growth pattern can be described using the StranskiKrastanov model ${ }^{46}$.

We analyzed the surfaces of as-received, $\mathrm{HNO}_{3}$ acid etched, $1000^{\circ} \mathrm{C}$ annealed, and $1100^{\circ} \mathrm{C}$ treated (melted) samples. Defect lines developed during the manufacturing process and $\mathrm{HNO}_{3}$ acid treatment are clearly visible from the optical images (Figs. $5 \mathrm{a}$ and $b$ ). After annealing at $1000^{\circ} \mathrm{C}$, grain boundaries are more noticeable, while the intrinsic defect lines are less identifiable (Fig. 5c). A very smooth surface with almost no defects is observed for the melted copper (Fig. 5d). Root-mean-square (RMS) roughness values of the asreceived copper range from 112 to $258 \mathrm{~nm}$ in five $10 \times 10 \mu \mathrm{m}^{2}$ sized areas. After the $\mathrm{HNO}_{3}$ acid etching treatment, the roughness value decreases slightly to 90-194 $\mathrm{nm}$. Surface copper oxides (Raman peaks at $526 \mathrm{~cm}^{-1}$ and $617 \mathrm{~cm}^{-1}$ assigned to $\mathrm{Cu}_{2} \mathrm{O}^{48-50}$ ) in the asreceived copper can be largely eliminated by the $\mathrm{HNO}_{3}$ acid etching treatment (Fig. 5e). Annealing the $\mathrm{HNO}_{3}$ acid treated copper foil at $1000^{\circ} \mathrm{C}$ under $\mathrm{H}_{2} / \mathrm{Ar}$ flow effectively lowered the roughness value to 

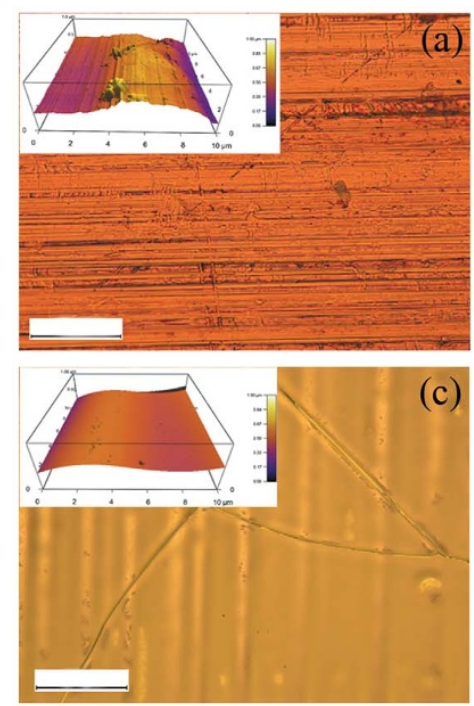
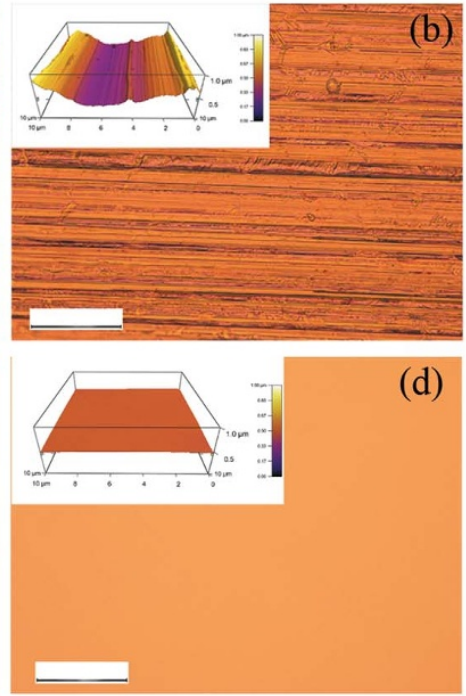

(b)

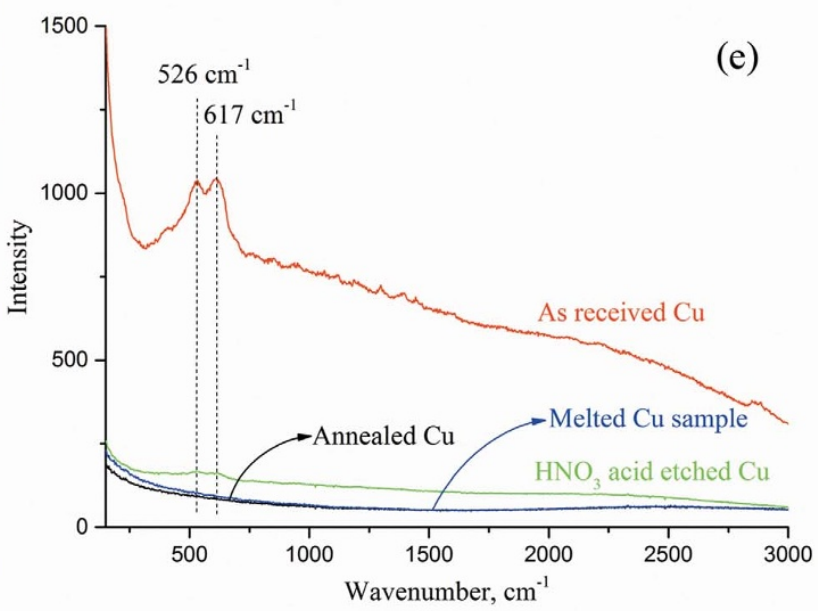

Figure $5 \mid$ Optical microscope images and Raman spectra of different copper substrates. (a) as-received, (b) $10 \% \mathrm{HNO} 3$ acid etched, (c) $1000^{\circ} \mathrm{C}$ annealed, and (d) $1100^{\circ} \mathrm{C}$ melted copper substrate. Scale bar is $50 \mu \mathrm{m}$. The inset in each of the images shows the three-dimensional (3D) AFM height profile across a $10 \times 10 \mu \mathrm{m} 2$ area. The color bar is within the 0 to $1 \mu \mathrm{m}$ range in all the insets. (e) Raman spectra of copper foil surfaces.

$7.023-10.21 \mathrm{~nm}$, with a further improvement to $4.681-4.761 \mathrm{~nm}$ for the melted copper. Raman analysis of the surfaces of the annealed solid and melted copper found no trace of copper oxide (Fig. 5e).

An interesting phenomenon was observed when the $h$-BNNS growth was carried out on solid copper at $1050^{\circ} \mathrm{C}$. At this temperature, initial $h$-BN domain formation is not found to occur preferentially on the original copper grain boundaries (Fig. 6). Furthermore, no adlayers on the initial domains (as at $1000^{\circ} \mathrm{C}$ ) were observed under SEM. With more ammonia borane $(50 \mathrm{mg})$, multilayered nanosheets with small $h$-BN adlayers on the top are observed. We believe that the copper surface undergoes a significant recrystallization process as the temperature approaches the melting point $\left(1083^{\circ} \mathrm{C}\right)$, resulting in a smoother surface and larger grains. At this temperature, the diffusion kinetic energy of $h$ - $\mathrm{BN}$ monomers is expected to be higher than that at $1000^{\circ} \mathrm{C}$, which is conducive to the formation of large $h$-BN domains. The insensitivity to high energy grain boundaries of the initial $h$-BN nucleation is not fully understood, however. Detailed theoretical and experimental investigations are required for this, which is beyond the scope of this study.

In conclusion, we have shown that several micron-sized single crystalline and mostly mono- and bi-layer $h$-BNNS can be grown on melted copper substrate, in contrast to largely inhomogeneous, 1 10 layer thick, nanocrystalline $h$-BNNS on the solid copper substrate. Possible $h$-BNNS growth processes on both solid and melted copper surfaces have been discussed. The smooth and nearly defect-free surface of the melted copper is believed to play the key role in the growth of high quality $h$-BNNS. This advantage is likely to work well with different precursors, such as a simultaneous flow of diborane and ammonia, to grow large $h$-BNNS on melted copper.

\section{Methods}

Synthesis of $\boldsymbol{h}$-BNNS on copper substrate. Figure 7 shows a schematic illustration of an APCVD system used for the growth of $h$-BNNS. A piece of copper foil $(0.25 \mathrm{~mm}$ thick, $99.98 \%$ purity) was pretreated in $10 \% \mathrm{HNO}_{3}$ solution and blow dried in $\mathrm{N}_{2}$ steam before being transferred into a quartz tube. Prior to heating, the whole system was evacuated and backfilled three times with a $10 \% \mathrm{H}_{2} / \mathrm{Ar}$ (volume percentage) gas mixture to remove air and moisture from the system. An annealing treatment was then carried out at $1000^{\circ} \mathrm{C}$ and $1100^{\circ} \mathrm{C}$ for the solid and melted copper substrates, respectively, for $40 \mathrm{~min}$ under $500 \mathrm{sccm} 10 \% \mathrm{H}_{2} / \mathrm{Ar}$ gas mixture flow to remove residual surface oxides and contamination. After the initial annealing period, growth of $h$-BNNS was continued for one hour by flowing the gaseous thermal decomposition products of ammonia borane at $110^{\circ} \mathrm{C}$ over the copper substrate (see details in Supplementary Information). A flow rate of $100 \mathrm{sccm}$ of $10 \% \mathrm{H}_{2} / \mathrm{Ar}$ carrier gas was used for this purpose. Under high carrier gas flow rates, for example, $200 \mathrm{sccm}, h$-BN nucleation was low, and highly discontinuous and inhomogeneous $h$-BNNS was observed (see Supplementary Information Fig. S7). Tungsten foil was used to support the melted copper, since it facilitates sufficient wetting of the melted surface. At the end of the growth, the furnace was quickly cooled down to room temperature by lifting the top cover of the furnace. Initially, $100 \mathrm{mg}$ of ammonia borane was used to compare the $h$-BNNS morphology, thickness, and crystallinity on both substrates. Growth processes of $h$-BNNS on those two substrates were then studied by varying the amounts of ammonia borane.

Transferring $\boldsymbol{h}$-BNNS to different substrates. $h$-BNNS was transferred to $285 \mathrm{~nm}$ $\mathrm{SiO}_{2} / \mathrm{Si}$ wafers and holey carbon TEM grids using a PMMA aided transfer process ${ }^{51}$ 3\% PMMA in chlorobenzene solution was spin coated at $4000 \mathrm{rpm}$ for 30 seconds on top of the $h$-BNNS grown on the copper surface. The samples were then dried for one
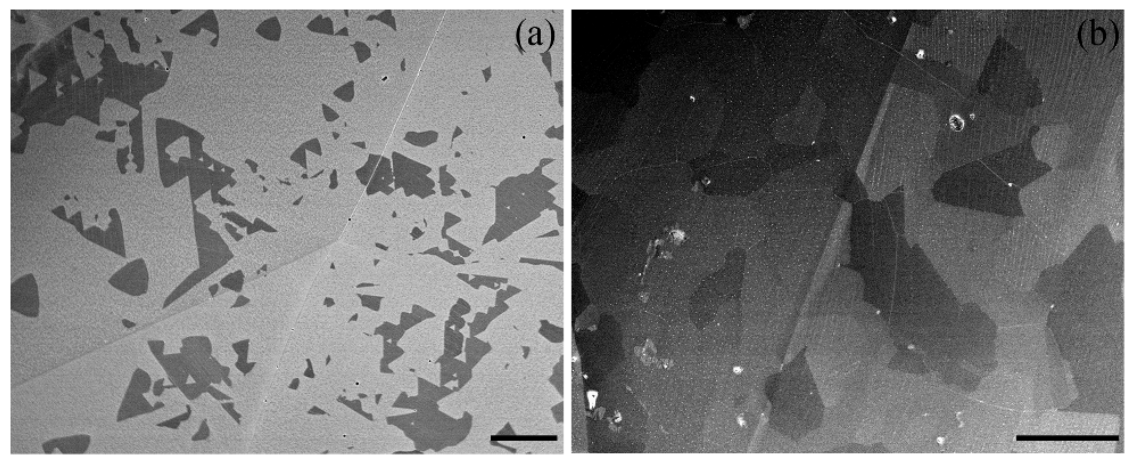

Figure 6 SEM images of h-BNNS grown at $1050^{\circ} \mathrm{C}$. (a) No preferential nucleation sites are observed for the initial h-BN domains. (b) Large and mixed multilayered h-BNNS with small adlayers on the top are found when more ammonia borane is used. Scale bar is $5 \mu \mathrm{m}$. 


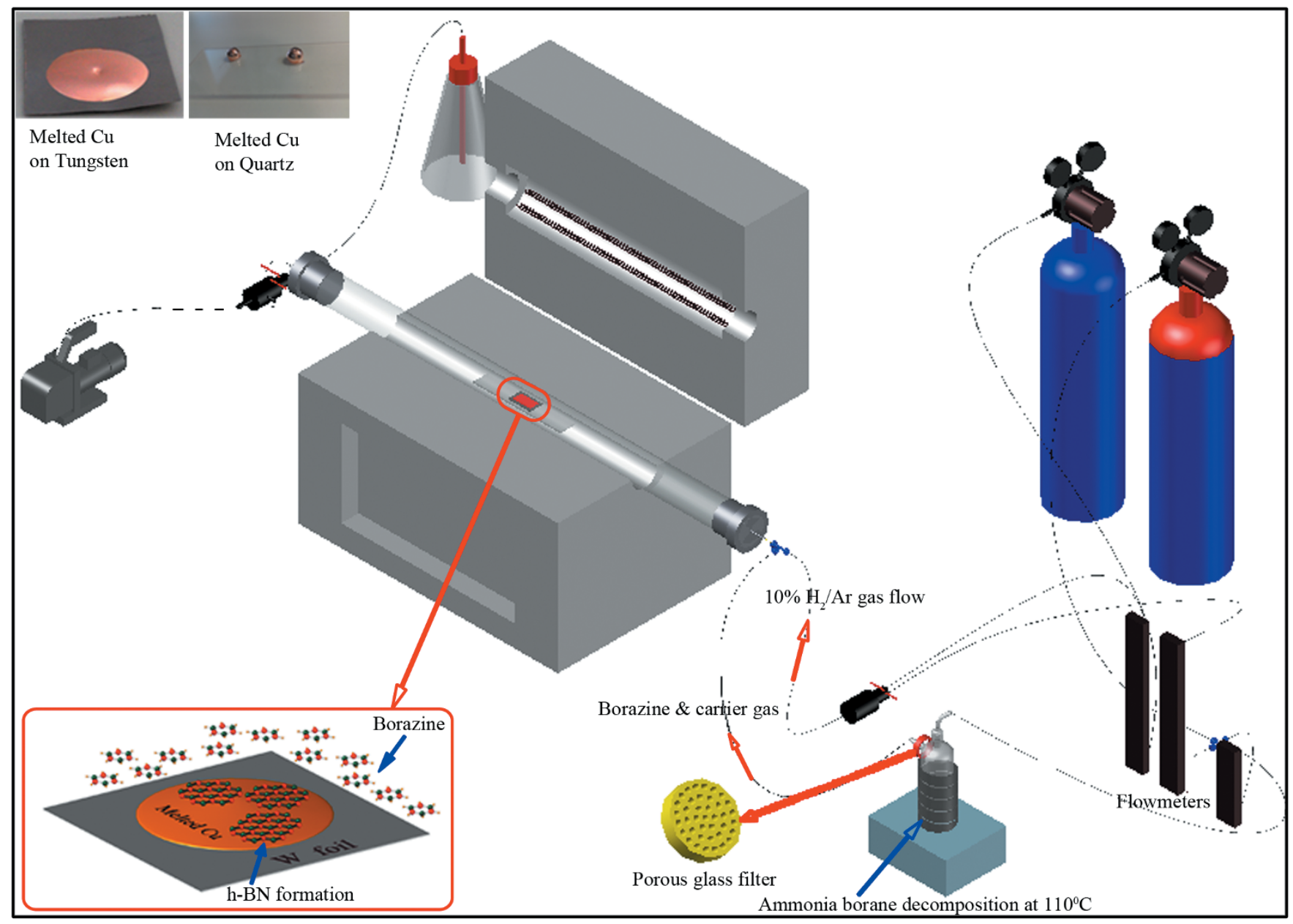

Figure $7 \mid$ Schematic illustration of an APCVD system. Volatile thermal decomposition products of ammonia borane were carried over the copper substrates by a 10\% H2/Ar gas mixture. For the melted copper, tungsten was used as the support due to its better wettability compared to the quartz slide.

hour before being left in a $0.05 \mathrm{M}$ ammonium persulfate etching solution overnight. The clean PMMA-coated $h$-BNNS was fished out on a $285 \mathrm{~nm} \mathrm{SiO} 2 / \mathrm{Si}$ wafer. The PMMA layer was removed using hot acetone and subsequent heat treatment at $350^{\circ} \mathrm{C}$ for two hours in $500 \mathrm{sccm} \mathrm{Ar} / \mathrm{H}_{2}$ flow. The adhesion of the $h$-BNNS to the substrate was strengthened by heating at $180^{\circ} \mathrm{C}$ for a few minutes.

Characterization. Optical microscope images were collected using a Leica DM6000 microscope. The surface roughness of the as-received and treated copper foils, together with the thickness of the $h$-BNNS, was acquired using an Asylum AFM MFP3D in the tapping mode. X-ray photoelectron spectra (XPS) were collected using a PHOIBOS 100 hemispherical analyzer. The X-ray excitation wavelength was provided by $\mathrm{Al} \mathrm{K} \alpha$ radiation with photon energy of $1486.6 \mathrm{eV}$. The XPS data were analyzed using CasaXPS software. The Shirley background function was used for B 1s and $\mathrm{N}$ 1s peak background correction. A 70\% Gaussian and 30\% Lorentzian combined function was used for peak-fitting analysis. Raman spectra were acquired directly on the copper foil substrates using a Horiba Raman JY HR 800 spectrometer. A $632.81 \mathrm{~nm}$ laser with a He-Ne source was used for the characterization. Raman spectra of $h$-BNNS on silicon wafers were obtained via a Renishaw inVia Raman spectrometer using a $514.0 \mathrm{~nm} \mathrm{Ar}^{+}$laser. Samples transferred to holey carbon TEM grids were examined in a probe-corrected JEOL ARM200F equipped with a cold field emission gun operated at an acceleration voltage of $80 \mathrm{kV}$. HAADF images were acquired with inner and outer collection angles of 50 to $180 \mathrm{mrad}$, respectively, ensuring $\mathrm{Z}$ contrast, while $\mathrm{BF}$ images were acquired with a maximum collection angle of $17 \mathrm{mrad}$. All images were acquired with a $20 \mu \mathrm{s}$ dwell time and convergence angle of $25 \mathrm{mrad}$, corresponding to a probe size of $\sim 1$ A and a current of $40 \mathrm{pA}$. Images were filtered by masking all visible reflections in the FFT as well as the central spot. $\{1120\}$ reflections were clearly observed in the FFT, which represents a minimum resolution of $0.145 \mathrm{~nm}$. EELS was performed using a GIF Quantum equipped in the same microscope. Field emission SEM images of $h$-BNNS on solid copper substrate were obtained using a scanning electron microscope (JEOL JSM-7500FA).

1. Yuzuriha, T. H. \& Hess, D. W. Structural and optical properties of plasmadeposited boron nitride films. Thin Solid Films 140, 199-207 (1986)
2. Watanabe, K., Taniguchi, T. \& Kanda, H. Direct-bandgap properties and evidence for ultraviolet lasing of hexagonal boron nitride single crystal. Nature Materials $\mathbf{3}$, 404-9 (2004)

3. Chen, W. et al. Hydrogenation: a simple approach to realize semiconductorhalf-metal- metal transition in boron nitride nanoribbons. Journal of the American Chemical Society 132, 1699-1705 (2010).

4. Tang, Q., Bao, J., Li, Y., Zhou, Z. \& Chen, Z. Tuning band gaps of BN nanosheets and nanoribbons via interfacial dihalogen bonding and external electric field. Nanoscale 6, 8624-34 (2014).

5. Liu, Z. et al. Ultrathin high-temperature oxidation-resistant coatings of hexagonal boron nitride. Nature Communications 4, 2541 (2013).

6. Liu, L. et al. Graphene oxidation: thickness-dependent etching and strong chemical doping. Nano Letters 8, 1965-70 (2008).

7. Rand, M. J. \& Roberts, J. F. Preparation and Properties of Thin Film Boron Nitride. Journal of The Electrochemical Society 115, 423 (1968).

8. Hummers, W. S., Jr. \& Offeman, R. E. Preparation of Graphitic Oxide. J. Am. Chem. Soc 80, 1339 (1958).

9. Weitz, R. T. \& Yacoby, A. Graphene rests easy. Nature 5, 699-700 (2010).

10. Kim, K. K. et al. Synthesis and characterization of hexagonal boron nitride film as a dielectric layer for graphene devices. ACS Nano 6, 8583-90 (2012).

11. Liu, Z. et al. In-plane heterostructures of graphene and hexagonal boron nitride with controlled domain sizes. Nature Nanotechnology 8, 119-24 (2013).

12. Lee, G. et al. Flexible and Transparent MoS 2 Field- Effect Transistors on Hexagonal Boron-Graphene Heterostructures. ACS Nano 7, 7931-7936 (2013).

13. Britnell, L. et al. Electron tunneling through ultrathin boron nitride crystalline barriers. Nano Letters 12, 1707-10 (2012).

14. Novoselov, K. S. et al. Two-dimensional atomic crystals. Proceedings of the National Academy of Sciences of the United States of America 102, 10451-3 (2005).

15. Coleman, J. N. et al. Two-dimensional nanosheets produced by liquid exfoliation of layered materials. Science 331, 568-71 (2011).

16. Zeng, H. et al. "White Graphenes": Boron Nitride Nanoribbons via Boron Nitride Nanotube Unwrapping. Nano Letters 10, 5049-5055 (2010). 
17. Liu, F. et al. Cheap, Gram-Scale Fabrication of BN Nanosheets via Substitution Reaction of Graphite Powders and Their Use for Mechanical Reinforcement of Polymers. Sci. Rep. 4, 4211 (2014).

18. Sutter, P., Lahiri, J., Zahl, P., Wang, B. \& Sutter, E. Scalable synthesis of uniform few-layer hexagonal boron nitride dielectric films. Nano Letters 13, 276-281 (2013).

19. Jin, C., Lin, F., Suenaga, K. \& Iijima, S. Fabrication of a Freestanding Boron Nitride Single Layer and Its Defect Assignments. Physical Review Letters 102, 195505 (2009).

20. Nagashima, A., Tejima, N., Gamou, Y., Kawai, T. \& Oshima, C. Electronic dispersion relations of monolayer hexagonal boron nitride formed on the $\mathrm{Ni}(111)$ surface. Physical Review B 51, 4606-4613 (1995).

21. Song, L. et al. Large scale growth and characterization of atomic hexagonal boron nitride layers. Nano Letters 10, 3209-3215 (2010).

22. Shi, Y. et al. Synthesis of few-layer hexagonal boron nitride thin film by chemical vapor deposition. Nano Letters 10, 4134-4139 (2010).

23. Gao, Y. et al. Repeated and controlled growth of monolayer, bilayer and few-layer hexagonal boron nitride on Pt foils. ACS Nano 7, 5199-5206 (2013).

24. Tay, R. Y. et al. Growth of Large Single-Crystalline Two-Dimensional Boron Nitride Hexagons on Electropolished Copper. Nano Letters 14, 839-846 (2014).

25. Kim, G. et al. Growth of high-crystalline, single-layer hexagonal boron nitride on recyclable platinum foil. Nano Letters 13, 1834-1839 (2013).

26. Kim, K. K. et al. Synthesis of monolayer hexagonal boron nitride on $\mathrm{Cu}$ foil using chemical vapor deposition. Nano Letters 12, 161-166 (2012).

27. Ismach, A. et al. Toward the controlled synthesis of hexagonal boron nitride films. ACS Nano 6, 6378-6385 (2012).

28. Tang, Q. \& Zhou, Z. Graphene-analogous low-dimensional materials. Progress in Materials Science 58, 1244-1315 (2013)

29. Auwa, W., Suter, H. U., Sachdev, H. \& Greber, T. Synthesis of One Monolayer of Hexagonal Boron Nitride on $\mathrm{Ni}(111)$ from B-Trichloroborazine $(\mathrm{ClBNH})_{3}$. Chem. Mater. 16, 343-345 (2004).

30. Müller, F. et al. Epitaxial growth of hexagonal boron nitride on $\operatorname{Ag}(111)$. Physical Review B 82, 113406 (2010).

31. Geng, D. et al. Uniform hexagonal graphene flakes and films grown on liquid copper surface. Proceedings of the National Academy of Sciences of the United States of America 109, 7992-6 (2012).

32. Wu, Y. A. et al. Large single crystals of graphene on melted copper using chemical vapor deposition. ACS Nano 6, 5010-5017 (2012).

33. Gorbachev, R. V. et al. Hunting for monolayer boron nitride: optical and Raman signatures. Small 7, 465-8 (2011).

34. Lin, Y. \& Connell, J. W. Advances in 2D boron nitride nanostructures: nanosheets, nanoribbons, nanomeshes, and hybrids with graphene. Nanoscale 4, 6908-39 (2012).

35. Pakdel, A., Zhi, C., Bando, Y., Nakayama, T. \& Golberg, D. Boron nitride nanosheet coatings with controllable water repellency. ACS Nano 5, 6507-15 (2011).

36. Bresnehan, M. S. et al. Integration of Hexagonal Boron Nitride with Quasifreestanding Epitaxial Graphene: Toward Wafer-Scale, High-Performance Devices. ACS Nano 6, 5234-5241 (2012).

37. Kelly, B. T. The anisotropic thermal expansion of boron nitride - II. Interpretation by the semi-continuum model. Philosophical Magazine 32, 859-867 (1975).

38. Krivanek, O. L. et al. Atom-by-atom structural and chemical analysis by annular dark-field electron microscopy. Nature 464, 571-4 (2010).

39. Chopra, N. G. et al. Boron nitride nanotubes. Science 269, 966-7 (1995).

40. Lee, G. et al. High-strength chemical-vapor-deposited graphene and grain boundaries. Science 340, 1073-6 (2013).

41. Huang, P. Y. et al. Grains and grain boundaries in single-layer graphene atomic patchwork quilts. Nature 469, 389-392 (2011).

42. Hao, Y. et al. The role of surface oxygen in the growth of large single-crystal graphene on copper. Science 342, 720-3 (2013).
43. Mohsin, A. et al. Synthesis of millimeter-size hexagon-shaped graphene single crystals on resolidified copper. ACS Nano 7, 8924-8931 (2013).

44. Sutter, P. \& Sutter, E. Thickness determination of few-layer hexagonal boron nitride films by scanning electron microscopy and Auger electron spectroscopy. APL Materials 092502, 1-7 (2014).

45. Li, Q. et al. Growth of adlayer graphene on $\mathrm{Cu}$ studied by carbon isotope labeling. Nano Letters 13, 486-490 (2013).

46. Venables, J. A. Introduction to surface and thin film processes. (Cambridge University Press, New York: 2000).

47. Wu, T. et al. Triggering the Continuous Growth of Graphene Toward MillimeterSized Grains. Advanced Functional Materials 23, 198-203 (2013).

48. Niaura, G. Surface-enhanced Raman spectroscopic observation of two kinds of adsorbed $\mathrm{OH}-$ ions at copper electrode. Electrochimica Acta 45, 3507-3519 (2000).

49. Chan, H. Y. H., Takoudis, C. G. \& Weaver, M. J. Oxide Film Formation and Oxygen Adsorption on Copper in Aqueous Media As Probed by SurfaceEnhanced Raman Spectroscopy. The Journal of Physical Chemistry B 103 357-365 (1999)

50. Hawaldar, R. et al. Large-area high-throughput synthesis of monolayer graphene sheet by Hot Filament Thermal Chemical Vapor Deposition. Sci. Rep. 2, 682 (2012).

51. Suk, J. W. et al. Transfer of CVD-grown monolayer graphene onto arbitrary substrates. ACS Nano 5, 6916-24 (2011).

\section{Acknowledgments}

Z.H. is the recipient of an Australian Research Council Discovery Early Career Research Award (project number DE120101496). Financial support through a University of Wollongong Near Miss Grant (2013), and a Collaborative Research Grant from the Australian Institute for Innovative Materials at UOW are acknowledged. This research used equipment funded by an Australian Research Council (ARC) Linkage, Infrastructure, Equipment and Facilities (LIEF) grant (LE120100104) located at the UOW Electron Microscopy Centre. The access to facilities at the Institute for Superconducting and Electronic Materials at UOW is appreciated.

\section{Author contributions}

M.H.K. and Z.H. designed the experiment and co-wrote the paper. M.H.K. performed the CVD growth and most of the characterization work (Optical, AFM, Raman, and SEM). G.C. conducted the TEM studies. F.X., Z.C., P.M. and H.K.L. participated in discussions and writing. All authors have given approval to the final version of the manuscript.

\section{Additional information}

Supplementary information accompanies this paper at http://www.nature.com/ scientificreports

Competing financial interests: The authors declare no competing financial interests.

How to cite this article: Khan, M.H. et al. Synthesis of Large and Few Atomic Layers of Hexagonal Boron Nitride on Melted Copper. Sci. Rep. 5, 7743; DOI:10.1038/srep07743 (2015)

This work is licensed under a Creative Commons Attribution-NonCommercialShareAlike 4.0 International License. The images or other third party material in this article are included in the article's Creative Commons license, unless indicated otherwise in the credit line; if the material is not included under the Creative Commons license, users will need to obtain permission from the license holder in order to reproduce the material. To view a copy of this license, visit http:// creativecommons.org/licenses/by-nc-sa/4.0/ 
SUBJECT AREAS: $\quad$ ERRATUM: Synthesis of Large and Few Atomic Layers of Hexagonal Boron MATERIALS CHEMISTRY Nitride on Melted Copper

NANOSCALE MATERIALS

Majharul Haque Khan, Zhenguo Huang, Feng Xiao, Gilberto Casillas, Zhixin Chen, Paul J. Molino \& Hua Kun Liu

SCIENTIFIC REPORTS:

$5: 7743$

DOI: $10.1038 /$ srep07743

(2015)

In this Article, the Figure labels were omitted from Figures 1-3. The correct Figures 1-3 appear below as Figure 1, Figure 2 and Figure 3 respectively.

There is also a typographical error in the Results section.

Published: 13 January 2015

Updated: 27 March 2015

"The SAED patterns show several sets of hexagonal patterns, indicating the polycrystalline nature of the sheets within the SAED zone $\left(800 \mathrm{~nm}^{2}\right)$ "

should read:

"The SAED patterns show several sets of hexagonal patterns, indicating the polycrystalline nature of the sheets within the SAED zone $(800 \mathrm{~nm})$ " 

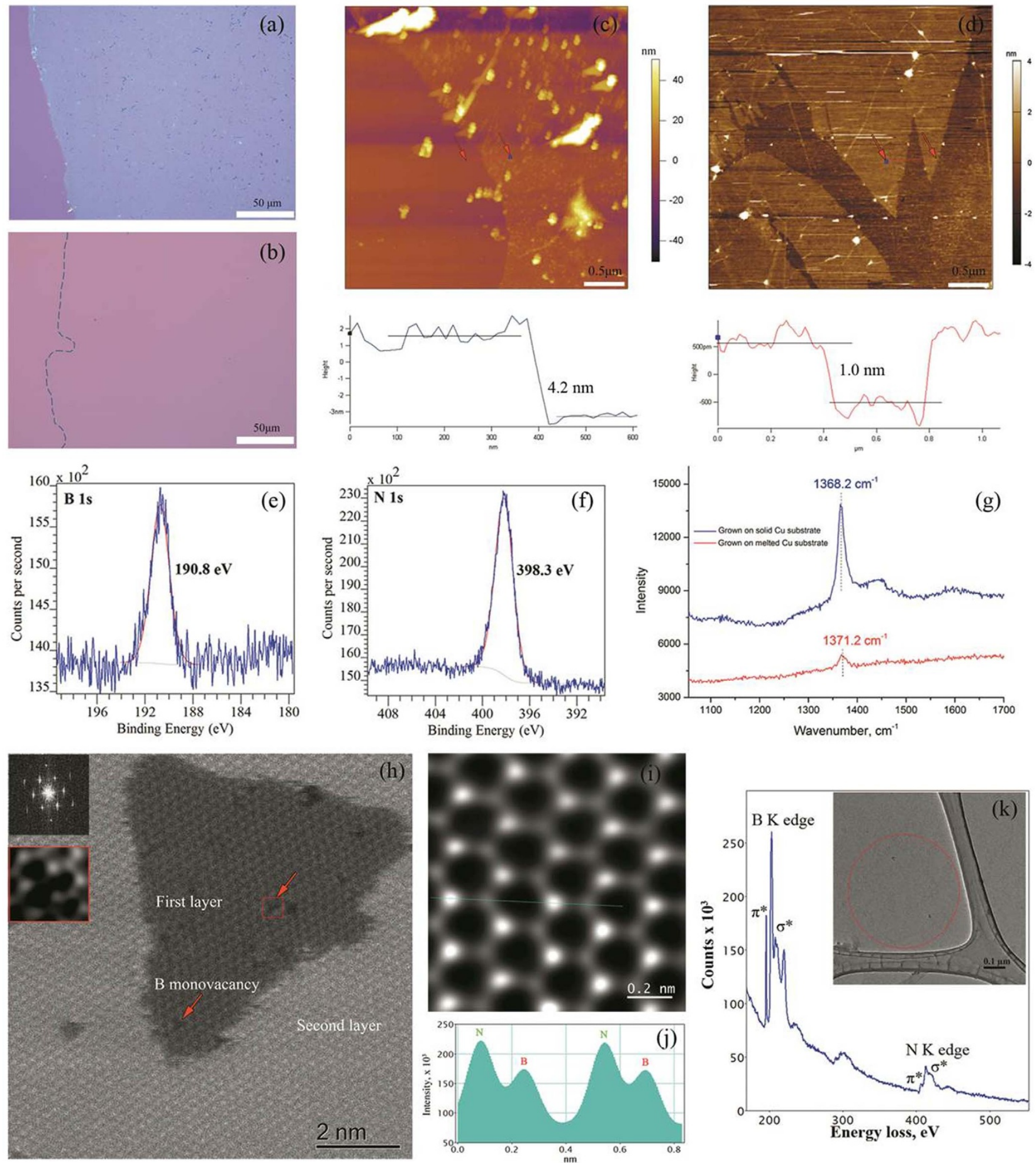

Figure $1 \mid$ 

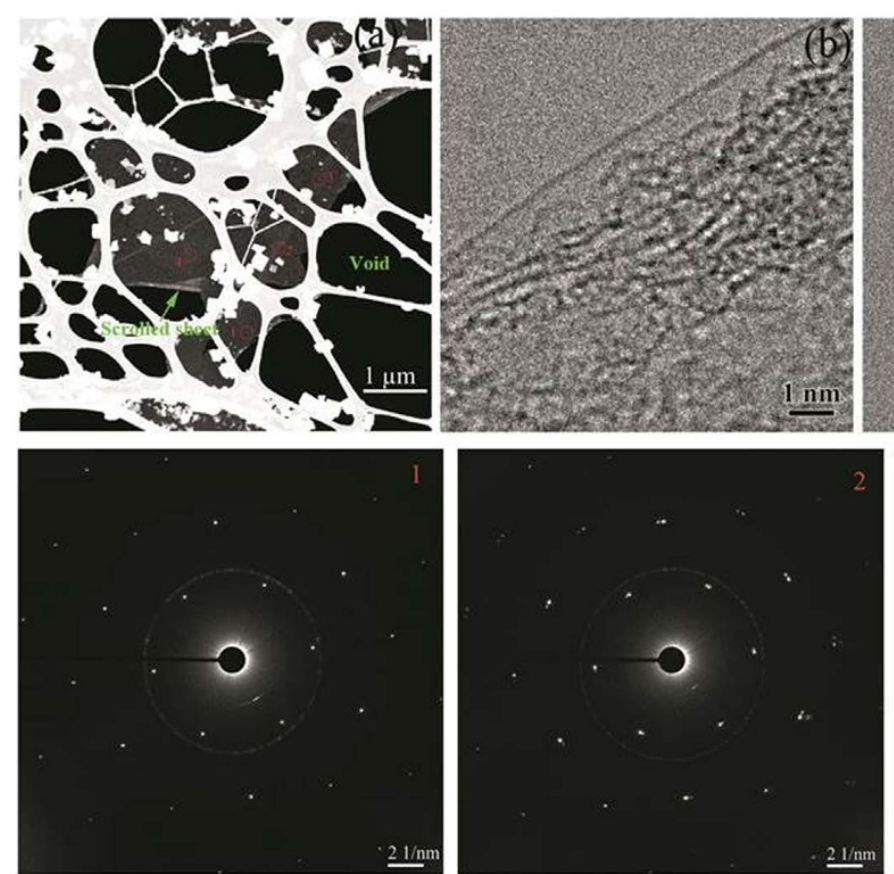

Figure 2
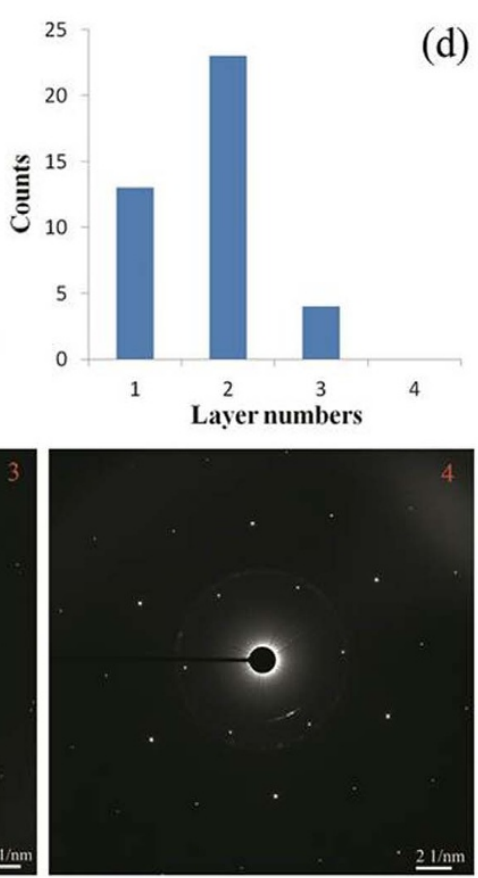

(d)
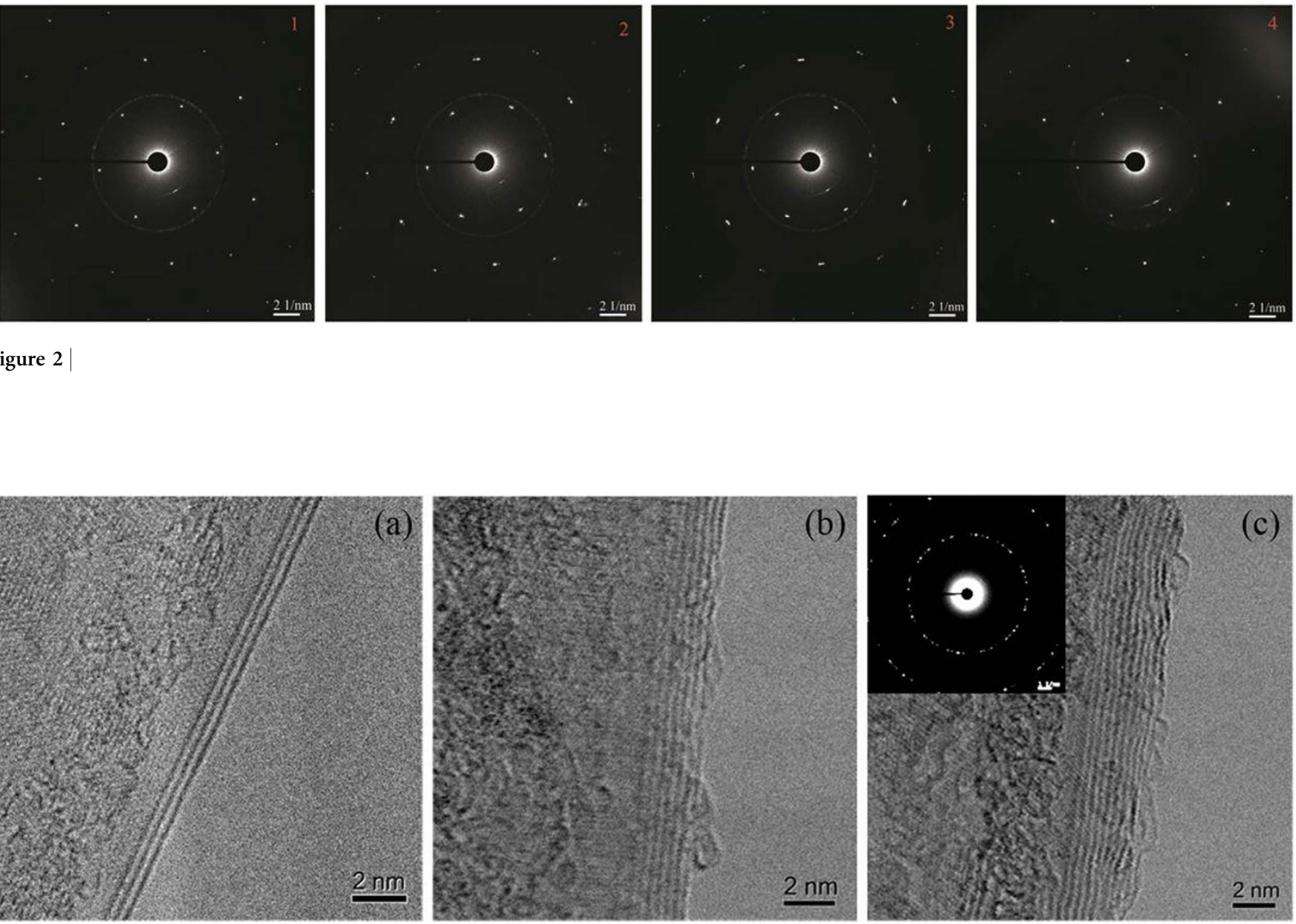

Figure 3 | 